\title{
The International Transporter Consortium: Summarizing Advances in the Role of Transporters in Drug Development
}

DOI:

10.1002/cpt.1224

Document Version

Accepted author manuscript

Link to publication record in Manchester Research Explorer

\section{Citation for published version (APA):}

Giacomini, K. M., Galetin, A., \& Huang, S. M. (2018). The International Transporter Consortium: Summarizing Advances in the Role of Transporters in Drug Development. Clinical Pharmacology \& Therapeutics, 104(5), 766771. https://doi.org/10.1002/cpt.1224

\section{Published in:}

Clinical Pharmacology \& Therapeutics

\section{Citing this paper}

Please note that where the full-text provided on Manchester Research Explorer is the Author Accepted Manuscript or Proof version this may differ from the final Published version. If citing, it is advised that you check and use the publisher's definitive version.

\section{General rights}

Copyright and moral rights for the publications made accessible in the Research Explorer are retained by the authors and/or other copyright owners and it is a condition of accessing publications that users recognise and abide by the legal requirements associated with these rights.

\section{Takedown policy}

If you believe that this document breaches copyright please refer to the University of Manchester's Takedown Procedures [http://man.ac.uk/04Y6Bo] or contact uml.scholarlycommunications@manchester.ac.uk providing relevant details, so we can investigate your claim.

\section{OPEN ACCESS}


The International Transporter Consortium: Summarizing advances in the role of transporters in drug development

Kathleen M. Giacomini ${ }^{1}$, Aleksandra Galetin ${ }^{2}$, and Shiew Mei Huang*3

1. Department of Bioengineering and Therapeutic Sciences, Schools of Pharmacy and Medicine, University of California, San Francisco, CA, USA; kathy.giacomini@ucsf.edu

2. Centre for Applied Pharmacokinetic Research, School of Health Sciences, The University of Manchester, Stopford Building, Oxford Road, Manchester M13 9PT, UK; aleksandra.galetin@manchester.ac.uk; +44-161-275-6886

3. Office of Clinical Pharmacology, Food and Drug Administration, 10903 New Hampshire Ave, Silver Spring, MD 20993, USA; shiewmei.huang@fda.hhs.gov; 301-796-1635

${ }^{*}$ Corresponding Author

Shiew Mei Huang, Ph.D.

Office of Clinical Pharmacology

Food and Drug Administration

10903 New Hampshire Ave

Silver Spring, MD 20993 USA

Email: shiewmei.huang@fda.hhs.gov; 301-796-1635

Word count: 1758; two figures; 20 references

\section{CONFLICT OF INTEREST}

The authors declared no competing interests for this work.

\section{FUNDING}

No funding was received for this work. 
The past few years have ushered in a wealth of new information on transporter biology, ranging from the publication of new crystal structures of the human glucose transporters (GLUTs; SLC2A2) (1) to the discovery of the mechanism by which the monocarboxylate transporter (SLC16A11) associates with type 2 diabetes (2), to a plethora of new therapies that target transporters in the treatment of both common and rare diseases. Complementing these fundamental advances in transporter biology, great strides have been made in our understanding of the role of transporters in the pharmacokinetics and pharmacodynamics of drugs, including transporter-mediated drug-drug interactions (DDIs), nutrient-drug interactions, and in drug efficacy and safety (3-5). The International Transporter Consortium (ITC) sponsored its third workshop as a preconference to the annual meeting of the American Society for Clinical Pharmacology and Therapeutics in the spring of 2017. The major goal of the preconference was to summarize recent advances in transporter pharmacology, which are of particular relevance to the development, approval and safe and effective use of prescription drugs. A range of topics including computational modeling of drug-transporter interactions and emerging transporters of clinical importance were presented, culminating in ten articles that are featured in this issue of Clinical Pharmacology and Therapeutics. These articles summarize recent advances in transporters in drug development and include a summary of a wealth of new information and a review of the recent relevant literature.

\section{Transporters of Emerging Clinical Importance}

Eight transporters have been the focus of previous ITC recommendations for prospective evaluation during drug development, including P-glycoprotein (P-pg, ABCB1); breast cancer resistance protein (BCRP, $A B C G 2)$; organic anion transporting polypeptide (OATP) 1B1 (SLCO1B1) and OATP1B3 (SLCO1B3); organic anion transporter (OAT) 1 (SLC22A6) and OAT3 (SLC22A8); organic cation transporter (OCT) 2 (SLC22A2), and multi-drug and toxin extrusion protein (MATE) 1 (SLC47A1) and to a lesser extent, MATE2-K (SLC47A2). Recommendations for retrospective evaluation, in which a drug developer may wish to examine the involvement of a particular transporter in the context of results obtained from a clinical study, has focused on other 
transporters. In making recommendations, the ITC has required a high level of evidence for the inclusion of a given transporter; most notably, substantial clinical evidence based on either DDI or genetic polymorphism studies that support the involvement of that transporter. In the current issue of Clinical Pharmacology and Therapeutics, Zamek-Gliszczynski and colleagues (6) extend the ITC recommendations for both prospective and retrospective evaluation. Prospectively, a wealth of new information has been published on the associations between genetic polymorphisms in OCT1 (SLC22A1) (7) and increases in plasma concentrations of drugs from a range of therapeutic classes. Thus, the ITC recommends testing of drugs as potential inhibitors of OCT1 prospectively during drug development. Considering recent clinical evidence supporting a role for OATP2B1 (SLCO2B1) in fruit juice-drug interactions and certain DDIs, OATP2B1 has been added to the list of transporters that should be evaluated retrospectively to explain a particular clinical finding that cannot be attributed to more common mechanisms (e.g., intestinal P-gp/BCRP, hepatic OATP1B1/3). The article extends previous ITC focus from DDIs exclusively to include vitamin-drug interactions. The authors suggest that assessing drug interactions with THTR2 (SLC19A3) in vitro may aid drug developers in predicting which drugs should be carefully monitored in susceptible populations for drug-induced thiamine deficiency (6).

Yee and colleagues (7) provide an update on polymorphisms that may be responsible for variation in drug absorption, disposition and therapeutic and adverse drug reactions. Genomewide and candidate gene association studies continue to support a strong role for coding region variants in BCRP and OATP1B1 as major determinants of interindividual variation in drug absorption, disposition and response. In addition, the article provides an excellent summary of recent candidate gene studies showing associations between common reduced function OCT1 polymorphisms and the pharmacokinetics and pharmacodynamics of various drugs.

\section{Clinical Evaluation of Transporter-mediated DDIs}

Zhang and colleagues (8) describe the FDA's continued recommendation of an integrated approach (in vitro, in vivo, and in silico) to assess DDI potential so that appropriate measures can 
be taken to minimize the risk of toxicities and ensure effectiveness. One of the challenges in the evaluation of transporter-mediated DDIs is the lack of specific substrates and inhibitors for various transporters, which makes extrapolation of DDI results from one drug pair to another difficult. This challenge was highlighted in the recently published FDA DDI guidances $(9,10)$ and also in the European Union and Japan guidance documents $(11,12)$. Therefore, the choice of transporter substrates or inhibitors for clinical DDI evaluation is typically based on the likelihood of co-administration (e.g., to obtain clinically relevant DDI information that can inform labeling regarding the management of a DDI).

Chu and colleagues (13) describe the emerging field of research identifying, characterizing and validating endogenous biomarkers of transporters. They describe the characteristics of an ideal biomarker, e.g., specificity for a transporter (similar to a drug probe), the importance of understanding the rate of formation and elimination of these endogenous molecules, and factors affecting their baseline stability or interindividual variability. Figure 1 identifies endogenous substrates as potential biomarkers for several hepatic and renal transporters. The article of Chu et al. provides both a comprehensive overview of the methods needed to identify and validate novel endogenous biomarkers and their limitations and challenges. In addition, the authors identify major gaps in biomarker discovery (e.g., for BCRP and P-gp) and summarize probe drugs (administered individually or as cocktails), for interrogating particular transporters in clinical studies.

Two additional commentaries in this issue provide useful advice on the design of clinical DDI studies focusing on the commonly prescribed anti-diabetic drug, metformin, and on the use of digoxin and dabigatran etexilate as probes for P-gp. The commentary by Zamek-Gliszczynski and colleagues (14) proposes a novel design of clinical DDI studies, which can support appropriate labeling of the use of new molecular entities, which are inhibitors of OCT/MATE when coadministered with metformin. The study design focuses on pharmacologic response endpoints (e.g., via an oral glucose tolerance test) and metformin renal clearance, in addition to changes in 
metformin systemic plasma concentrations. Chu and colleagues (15) highlight the use of dabigatran etexilate as a probe substrate for intestinal P-gp DDIs, noting that the compound is a prodrug and therefore does not interact with P-gp in other organs. The advantages and disadvantages of both digoxin and dabigatran etexilate as P-gp probes (microdose and/or standard dose) are discussed.

\section{Integrated Evaluation of Transporter Effects}

There is increasing clinical evidence of profound transporter-mediated DDIs at the tissue level that are not always supported by standard investigation of changes in the systemic exposure of a drug probe. Guo and colleagues (16) provide a critical overview of current advances in in vitro/preclinical models, imaging methods and physiologically-based pharmacokinetic (PBPK) modeling and simulation for estimation or prediction of tissue and intracellular drug concentrations, including organelle targeting (specifically lysosomes and mitochondria). The authors emphasize a need for integration of different approaches and propose a workflow in the context of drug development (Figure 2). The concept is supported by 23 PBPK modeling and simulation examples from the literature/regulatory submissions and three industrial case studies detailed in the article. These examples illustrate best practices in the verification of modelsimulated tissue exposure using pharmacologic response data (statins, simeprevir) and implementation of mechanistic liver and kidney models to simulate transporter-mediated disposition in the case of renal impairment (digoxin, OAT1 probes), hepatic impairment (obeticholic acid) or extrapolation across ethnic groups or diseased populations (letermovir). However, examples where quantitative PET (positron emission tomography)/MRI (magnetic resonance imaging) tissue data are used for development or verification of PBPK models are still sparse.

Schlessinger and colleagues (17) illustrate recent progress in molecular modeling of membrane transporters, including approaches focusing on the structures of transporters and transporter ligands. The authors provide current best practices in molecular modeling techniques and 
emphasize the importance of quality in vitro functional transporter data and crystal structures of relevant transporters for evaluation of model performance and recent advances in this area. 


\section{Disease Associated Changes in Transporter Expression and/or Activity}

Complex interplay of multiple processes and/or multiple organs involved in transporter-mediated disposition can be a challenge in defining the rate-determining step and consequences of perturbation of one or multiple mechanisms. These challenges are discussed from the in vitro/preclinical, imaging and PBPK modeling perspective (16), as well as disease-associated changes in transporter expression and/or activity $(18,19)$. Taskar and colleagues (18) discuss prospective evaluation of inhibition of the bile salt export pump (BSEP) as one of the mechanisms contributing to drug-induced liver injury (DILI). The authors propose a workflow for interpretation and mitigation of BSEP inhibition in drug discovery and/or early clinical development and propose more physiologically relevant cellular systems (e.g., sandwich cultured hepatocytes) to investigate the role of metabolites and complex interplay between BSEP and other bile acids transporters in DILI. Uncertainty in BSEP in vitro inhibition data and subsequent interpretation of such data in the context of in vivo exposure are critically discussed and highlight the necessity to increase our confidence in predicting drug tissue exposure (16). Recent quantitative systems toxicology efforts to integrate effects of multiple transporters and multiple DILI mechanisms (e.g., mitochondrial toxicity and oxidative stress) are encouraging, but are not widely adopted.

Increasing evidence exists about changes in transporter expression and activity as a result of acute and chronic disease and altered physiological states; these important aspects are summarized comprehensively in the manuscript by Evers and colleagues (19). An additional consideration is the potential effect on regulatory pathways of drug transporters and changes in transporter localization in response to disease, as illustrated by multidrug resistance-associated protein 2 (MRP2, ABCC2) redistribution from the plasma membrane to intracellular compartments in patients with nonalcoholic steatohepatitis. Knowledge and quantitative data on disease-related changes in transporter expression and/or activity are key to inform PBPK modeling and facilitate MIDD (model-informed drug development) approaches (20), to support individual dose optimization and, ultimately, improved effectiveness and product safety. 


\section{Conclusion:}

Alterations in transporter function due to various intrinsic factors (e.g., genetics, disease states) and extrinsic factors (concomitant drugs or nutrients) can lead to variability in the pharmacokinetics, pharmacodynamics, efficacy and safety of drugs in individual patients. The ITC articles in this special issue identified key transporters of clinical relevance that need to be evaluated either prospectively or retrospectively during drug development. Novel methodologies and best practices (in vitro, in vivo, in silico and their integrated application) have been presented in the evaluation of these transporters. Although great advancements have been made in our understanding of factors affecting transporter function and clinical relevance, challenges remain, and the knowledge gaps must be addressed via collaborative efforts such as this international consortium.

\section{DISCLAIMER}

The contents of this article reflect the views of the authors and should not be construed to represent the FDA's views or policies. No official support or endorsement by the FDA is intended or should be inferred. The mention of commercial products, their sources, or their use in connection with material reported herein is not to be construed as either an actual or implied endorsement of such products by the FDA.

\section{ACKNOWLEDGEMENT}

The authors thank Drs. Xiaoyan Chu (Merck) and Lei Zhang (FDA) for providing Figure 1. 
Figure legends:

Figure 1. Potential endogenous biomarkers for several hepatic and renal transporters. [adapted/modified from Chu et al. (13)].

The endogenous substrates with clinical DDI evaluation of potent transporter inhibitor(s) have been included into this figure as potential endogenous biomarkers. Coproporphyrin I (CPI) and CPIII, glycochenodeoxycholate-3-O-sulfate (GCDCA-S), conjugated and unconjugated bilirubin (CB and UCB) are potential endogenous biomarkers for OATP1B1 and -1B3. Hexadecanedioate (HDA) and tetradecanedioate (TDA) are potential biomarkers for OATP1B1 (they are not substrates for OATP1B3). N1-methylnicotinamide (NMN) and creatinine are potential endogenous biomarkers of OCT2/MATE1/2K. Taurine is a potential endogenous biomarker for OAT1. $6 \beta$-hydroxycortisol (6ßHC) and GCDCA-S are potential endogenous biomarkers of OAT3.

a: Potential endogenous biomarkers with relatively higher selectivity and/or sensitivity for clinical evaluation based on current literature data.

b. Hexadecanedioate (HDA) and tetradecanedioate (TDA) are potential biomarkers for OATP1B1 (they are not substrates for OATP1B3).

N.I.: None identified

Figure 2. Integrated workflow to illustrate in vitro/preclinical and clinical data generation and modeling considerations to identify, characterize and predict human pharmacokinetics in plasma and tissues for molecules where transporter plays an important role. [adapted/modified from Guo et al. (16)].

a See case study 1 in ref. (16), 'bee case study 2 in ref. (16), 'See case study 3 in ref. (16), d Reference (13), ${ }^{\mathrm{e}}$ Reference (7) 


\section{References:}

(1) Deng, D. et al. Crystal structure of the human glucose transporter GLUT1. Nature 510, 121-5 (2014).

(2) Rusu, V. et al. Type 2 Diabetes Variants Disrupt Function of SLC16A11 through Two Distinct Mechanisms. Cell 170, 199-212 e20 (2017).

(3) Zamek-Gliszczynski, M.J. et al. ITC recommendations for transporter kinetic parameter estimation and translational modeling of transport-mediated PK and DDIs in humans. Clin Pharmacol Ther 94, 64-79 (2013).

(4) Galetin, A., Zhao, P. \& Huang, S.M. Physiologically Based Pharmacokinetic Modeling of Drug Transporters to Facilitate Individualized Dose Prediction. J Pharm Sci 106, 2204-8 (2017).

(5) Liang, X. et al. Metformin Is a Substrate and Inhibitor of the Human Thiamine Transporter, THTR-2 (SLC19A3). Mol Pharm 12, 4301-10 (2015).

(6) Zamek-Gliszczynski, M.J. et al. Transporters in Drug Development: 2018 ITC Recommendations for Emerging Transporters of Clinical Importance. Clin Pharmacol Ther, (2018).

(7) Yee, S.W. et al. Influence of Transporter Polymorphisms on Drug Disposition and Response: A Perspective From the International Transporter Consortium. Clin Pharmacol Ther, (2018).

(8) Zhang, L., Huang, S.-M., Reynolds, K., Madabushii, R. \& Zineh, I. Transporters in Drug Development: Scientific and Regulatory Considerations. . Clin Pharmacol Ther, (2018).

(9) US Department of Health and Human Services, Food and Drug Administration, Center for Drug Evaluation and Research (CDER) Draft guidance/guidance for industry. In Vitro Metabolism- and Transporter- Mediated Drug-Drug Interaction Studies Guidance for Industry https://wwwfdagov/downloads/Drugs/Guidances/UCM581965pdf.

When finalized, the guidance will be available at the following link: https://www.fda.gov/Drugs/GuidanceComplianceRegulatorylnformation/Guidances/ucm0 64982.htm (2017).

(10) US Department of Health and Human Services, Food and Drug Administration, Center for Drug Evaluation and Research (CDER) Draft guidance/guidance for industry. Clinical Drug Interaction Studies - Study Design, Data Analysis, and Clinical Implications Guidance for Industry.

https://wwwfdagov/downloads/Drugs/GuidanceComplianceRegulatory/nformation/Guidan ces/UCM292362pdf When finalized, the guidance will be available at the following link: https://www.fda.gov/Drugs/GuidanceComplianceRegulatorylnformation/Guidances/ucm0 $\underline{6}$ (2017).

(11) European Medicines Agency, Guideline on the investigation of drug interactions. http://www.ema.europa.eu/docs/en GB/document library/Scientific guideline/2012/07/W C500129606.pdf (2013).

(12) Ministry of Health, Labour and Welfare, Japan. Guideline of drug interaction studies for drug development and appropriate provision of information. http://www.pmda.go.jp/files/000206158.pdf. (2014).

(13) Chu, X. et al. Clinical Probes and Endogenous Biomarkers as Substrates for Transporter DDI Evaluation: Perspectives from the International Transporter Consortium Clin Pharmacol Ther, (2018).

(14) Zamek-Gliszczynski, M.J. et al. ITC Commentary on Metformin Clinical Drug-Drug Interaction Study Design That Enables an Efficacy- and Safety-Based Dose Adjustment Decision. Clin Pharmacol Ther, (2018).

(15) Chu, X., Galetin, A., Zamek-Gliszczynski, M.J., Zhang, L. \& Tweedie, D. Dabigatran Etexilate and Digoxin: Comparison as Clinical Probe Substrates for P-gp Inhibition Evaluation. Clin Pharmacol Ther, (2018).

(16) Guo, Y. et al. Advancing Predictions of Tissue and Intracellular Drug Concentrations Using In Vitro, Imaging and PBPK Modeling Approaches Clin Pharmacol Ther, (2018). 
(17) Schlessinger, A., Welch, M.A., Korzekwa, K., Swaan, P.W. \& Matsson, P. Molecular Modeling of Drug-Transporter Interactions-an International Transporter Consortium Perspective. Clin Pharmacol Ther, (2018).

(18) Taskar, K.S. et al. Can BSEP Inhibition Testing In Drug Discovery And Development Reduce Liver Injury Risk?: An International Transporter Consortium Perspective. Clin Pharmacol Ther (2018).

(19) Evers, R. et al. Disease-Associated Changes in Drug Transporters May Impact the Pharmacokinetics and/or Toxicity of Drugs: A White Paper from the International Transporter Consortium. Clin Pharmacol Ther, (2018).

(20) US Food and Drug Administration Prescription Drug User Fee Act. PDUFA VI Fiscal Years 2018-2022.

https://www.fda.gov/forindustry/userfees/prescriptiondruguserfee/ucm446608.htm. 



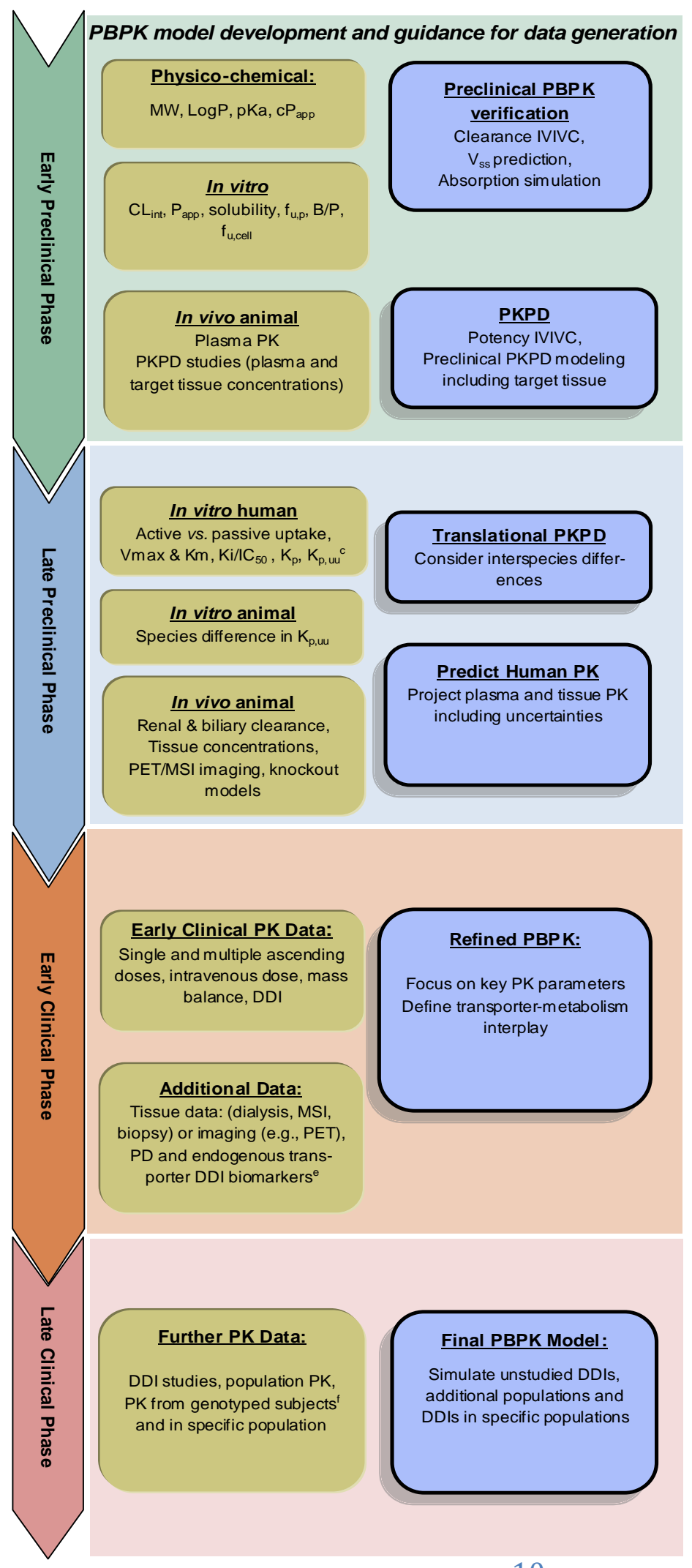

